\title{
Front Matter: Volume 8504
}

, "Front Matter: Volume 8504," Proc. SPIE 8504, X-Ray Free-Electron Lasers: Beam Diagnostics, Beamline Instrumentation, and Applications, 850401 (13 November 2012); doi: 10.1117/12.2012088

SPIE Event: SPIE Optical Engineering + Applications, 2012, San Diego, California, SPIE. United States 


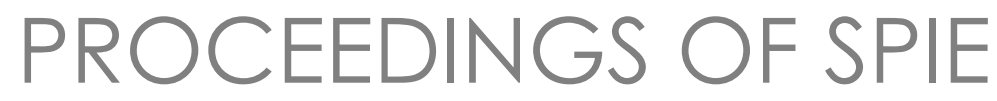

\title{
X-Ray Free-Electron Lasers: Beam Diagnostics, Beamline Instrumentation, and Applications
}

\author{
Stefan P. Moeller \\ Makina Yabashi \\ Stefan P. Hau-Riege \\ Editors
}

13-16 August 2012

San Diego, California, United States

Sponsored and Published by

SPIE 
The papers included in this volume were part of the technical conference cited on the cover and title page. Papers were selected and subject to review by the editors and conference program committee. Some conference presentations may not be available for publication. The papers published in these proceedings reflect the work and thoughts of the authors and are published herein as submitted. The publisher is not responsible for the validity of the information or for any outcomes resulting from reliance thereon.

Please use the following format to cite material from this book:

Author(s), "Title of Paper," in X-Ray Free-Electron Lasers: Beam Diagnostics, Beamline Instrumentation, and Applications, edited by Stefan P. Moeller, Makina Yabashi, Stefan P. Hau-Riege, Proceedings of SPIE Vol. 8504 (SPIE, Bellingham, WA, 2012) Article CID Number.

ISSN: 0277-786X

ISBN: 9780819492210

Published by

SPIE

P.O. Box 10, Bellingham, Washington $98227-0010$ USA

Telephone +1 3606763290 (Pacific Time) · Fax +1 3606471445

SPIE.org

Copyright () 2012, Society of Photo-Optical Instrumentation Engineers.

Copying of material in this book for internal or personal use, or for the internal or personal use of specific clients, beyond the fair use provisions granted by the U.S. Copyright Law is authorized by SPIE subject to payment of copying fees. The Transactional Reporting Service base fee for this volume is $\$ 18.00$ per article (or portion thereof), which should be paid directly to the Copyright Clearance Center (CCC), 222 Rosewood Drive, Danvers, MA 01923. Payment may also be made electronically through CCC Online at copyright.com. Other copying for republication, resale, advertising or promotion, or any form of systematic or multiple reproduction of any material in this book is prohibited except with permission in writing from the publisher. The CCC fee code is 0277-786X/12/\$18.00.

Printed in the United States of America.

Publication of record for individual papers is online in the SPIE Digital Library.

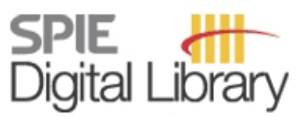

SPIEDigitalLibrary.org

Paper Numbering: Proceedings of SPIE follow an e-First publication model, with papers published first online and then in print and on CD-ROM. Papers are published as they are submitted and meet publication criteria. A unique, consistent, permanent citation identifier (CID) number is assigned to each article at the time of the first publication. Utilization of CIDs allows articles to be fully citable as soon as they are published online, and connects the same identifier to all online, print, and electronic versions of the publication. SPIE uses a six-digit CID article numbering system in which:

- The first four digits correspond to the SPIE volume number.

- The last two digits indicate publication order within the volume using a Base 36 numbering

system employing both numerals and letters. These two-number sets start with $00,01,02,03,04$, $05,06,07,08,09,0 A, 0 B \ldots 0 Z$, followed by 10-1Z, 20-2Z, etc.

The CID Number appears on each page of the manuscript. The complete citation is used on the first page, and an abbreviated version on subsequent pages. Numbers in the index correspond to the last two digits of the six-digit CID Number. 


\section{Contents}

ix Conference Committee

xi Introduction

\section{DIAGNOSTICS I}

850403 Profiling structured beams using injected aerosols [8504-4]

N. D. Loh, D. Starodub, SLAC National Accelerator Lab. (United States); L. Lomb,

Max-Planck-Institut für medizinische Forschung (Germany); C. Y. Hampton, SLAC National Accelerator Lab. (United States); A. V. Martin, Deutsches Elektronen-Synchrotron (Germany); R. G. Sierra, SLAC National Accelerator Lab. (United States); A. Barty, Deutsches Elektronen-Synchrotron (Germany); A. Aquila, J. Schulz, European XFEL GmbH (Germany); J. Steinbrener, R. L. Shoeman, S. Kassemeyer, Max-Planck-Institut für medizinische Forschung (Germany); C. Bostedt, J. Bozek, SLAC National Accelerator Lab. (United States); S. W. Epp, B. Erk, Max-Planck-Arbeitsgruppen für strukturelle Molekularbiologie (Germany); R. Hartmann, PNSensor GmbH (Germany); D. Rolles, A. Rudenko, B. Rudek, L. Foucar, Max-Planck-Arbeitsgruppen für strukturelle Molekularbiologie (Germany); N. Kimmel, G. Weidenspointner, G. Hauser, Max-PlanckInstitut Halbleiterlabor (Germany); P. Holl, PNSensor GmbH (Germany); E. Pedersoli, Sincrotrone Trieste S.C.p.A. (Italy); M. Liang, Deutsches Elektronen-Synchrotron (Germany); M. S. Hunter, Lawrence Livermore National Lab. (United States); L. Gumprecht, Deutsches Elektronen-Synchrotron (Germany); N. Coppola, European XFEL GmbH (Germany); C. Wunderer, H. Graafsman, Deutsches Elektronen-Synchrotron (Germany); F. R. N. C. Maia, Lawrence Berkeley National Lab. (United States); T. Ekeberg, M. Hantke, Uppsala Univ. (Sweden); H. Fleckenstein, Deutsches Elektronen-Synchrotron (Germany); H. Hirsemann (Germany); K. Nass, T. A. White, Deutsches Elektronen-Synchrotron (Germany); H. J. Tobias, Cornell Univ. (United States); G. R. Farquar, W. Benner, S. Hau-Riege, Lawrence Livermore National Lab. (United States); C. Reich, A. Hartmann, H. Soltau, PNSensor GmbH (Germany); S. Marchesini, Lawrence Berkeley National Lab. (United States); S. Bajt, M. Barthelmess, Deutsches Elektronen-Synchrotron (Germany); L. Strueder, Max-Planck-Institut Halbleiterlabor (Germany); J. Ullich, Max-Planck-Institut für Kernphysik (Germany); P. Bucksbaum, K. O. Hodgson, SLAC National Accelerator Lab. (United States); M. Frank, Lawrence Livermore National Lab. (United States); I. Schlichting, Max-Planck-Institut für medizinische Forschung (Germany); H. N. Chapman, Deutsches Elektronen-Synchrotron (Germany); M. J. Bogan, SLAC National Accelerator Lab. (United States)

850404 The photon beam transport and diagnostics system at FERMI@Elettra, the Italian seeded FEL source: commissioning experience and most recent results [8504-2] M. Zangrando, Lab. Nazionale TASC (Italy) and Sincrotrone Trieste S.C.p.A. (Italy); A. Abrami, Sincrotrone Trieste S.C.P.A. (Italy); D. Cocco, SLAC National Accelerator Lab. (United States); C. Fava, S. Gerusina, R. Gobessi, N. Mahne, E. Mazzucco, L. Raimondi, L. Rumiz, C. Svetina, F. Parmigiani, Sincrotrone Trieste S.C.p.A. (Italy) 
850405 X-ray imaging and imaging spectroscopy of fusion plasmas and light-source experiments with spherical optics and pixel array detectors [8504-3]

K. W. Hill, M. Bitter, L. Delgado-Aparicio, N. A. Pablant, Princeton Plasma Physics Lab.

(United States); P. Beiersdorfer, Lawrence Livermore National Lab. (United States);

M. Sanchez del Rio, European Synchrotron Radiation Facility (France); L. Zhang, Institute of Plasma Physics (China)

\section{OPTICS I}

850407 Design of an x-ray split- and delay-unit for the European XFEL (Invited Paper) [8504-6] S. Roling, Westfälische Wilhelms-Univ Münster (Germany); L. Samoylova, European XFEL GmbH (Germany); B. Siemer, Westfälische Wilhelms-Univ. Münster (Germany); H. Sinn, European XFEL GmbH (Germany); F. Siewert, Helmholtz-Zentrum Berlin für Materialien und Energie GmbH (Germany); F. Wahlert, M. Wöstmann, H. Zacharias, Westfälische WilhelmsUniv. Münster (Germany)

850409 Mirror-based soft x-ray split-and-delay system for femtosecond pump-probe experiments at LCLS [8504-8]

B. F. Murphy, Western Michigan Univ. (United States); J.-C. Castagna, J. D. Bozek, SLAC National Accelerator Lab. (United States); N. Berrah, Western Michigan Univ. (United States)

\section{DETECTORS}

8504 OC The CSPAD megapixel x-ray camera at LCLS [8504-11]

P. Hart, S. Boutet, G. Carini, M. Dubrovin, B. Duda, D. Fritz, G. Haller, R. Herbst, S. Herrmann, C. Kenney, N. Kurita, H. Lemke, M. Messerschmidt, M. Nordby, J. Pines, D. Schafer, M. Swift, M. Weaver, G. Williams, D. Zhu, SLAC National Accelerator Lab. (United States);

N. Van Bakel, Nationaal Institute for Subatomic Physics (Netherlands); J. Morse, European Synchrotron Radiation Facility (France)

8504 OD Bunch by bunch beam monitoring in 3rd and 4th generation light sources by means of single crystal diamond detectors and quantum well devices [8504-12] M. Antonelli, M. Di Fraia, Univ. degli Studi di Trieste (Italy); A. Tallaire, J. Achard, LSPM, CNRS, Univ. Paris 13 (France); S. Carrato, Univ. degli Studi di Trieste (Italy); R. H. Menk, G. Cautero, D. Giuressi, W. H. Jark, Sincrotrone Trieste S.C.p.A. (Italy); G. Biasiol, Lab. Nazionale TASC (Italy); T. Ganbold, Lab. Nazionale TASC (Italy) and Univ. degli Studi di Trieste (Italy); K. Oliver, Diamond Detectors, Ltd. (United Kingdom); C. Callegari, M. Coreno, Sincrotrone Trieste S.C.p.A. (Italy) and CNR-IMIP (Italy); A. De Sio, E. Pace, Univ. degli Studi di Firenze (Italy) 
8504 OF Developing a platform for high-resolution phase contrast imaging of high pressure shock waves in matter [8504-14]

A. Schropp, Technische Univ. Dresden (Germany) and SLAC National Accelerator Lab. (United States); J. Patommel, F. Seiboth, Technische Univ. Dresden (Germany); B. Arnold, E. C. Galtier, H. J. Lee, B. Nagler, J. B. Hastings, SLAC National Accelerator Lab. (United States); C. G. Schroer, Technische Univ. Dresden (Germany)

8504 OG Resonant soft $x$-ray scattering endstation for time-resolved pump-probe measurements at LCLS [8504-16]

Y.-D. Chuang, D. Doering, A. G. Cruz, N. Tahir, N. C. Andresen, K. P. Chow, D. Contarato, C. L. Cummings, E. E. Domning, J. Joseph, J. S. Pepper, B. V. Smith, G. Zizka, Lawrence Berkeley National Lab. (United States); C. Ford, W.-S. Lee, M. Weaver, SLAC National Accelerator Lab. (United States); L. Patthey, Paul Scherrer Institut (Switzerland); J. Weizeowick, Argonne National Lab. (United States); P. Denes, Z. Hussain, Lawrence Berkeley National Lab. (United States)

$8504 \mathrm{OH}$ Modeling of XFEL induced ionization and atomic displacement in protein nanocrystals [8504-17]

C. Caleman, Deutsches Elektronen-Synchrotron (Germany) and Uppsala Univ (Sweden): N. Tîmneanu, Uppsala Univ. (Sweden); A. V. Martin, Deutsches Elektronen-Synchrotron (Germany) and Univ of Melbourne (Australia); T. A. White, Deutsches ElektronenSynchrotron (Germany); H. A. Scott, Lawrence Livermore National Lab. (United States); A. Barty, Deutsches Elektronen-Synchrotron (Germany); A. Aquila, Deutsches ElektronenSynchrotron (Germany) and European XFEL GmbH (Germany); H. N. Chapman, Deutsches Elektronen-Synchrotron (Germany) and Univ. Hamburg (Germany)

8504 Ol Hard $x$-ray delay line for $x$-ray photon correlation spectroscopy and jitter-free pump-probe experiments at LCLS [8504-34]

W. Roseker, Deutsches Elektronen-Synchrotron (Germany); S. Lee, Korea Research Institute of Standards and Science (Korea, Republic of); M. Walther, H. Schulte-Schrepping,

H. Franz, Deutsches Elektronen-Synchrotron (Germany); A. Gray, M. Sikorski, SLAC National Accelerator Lab. (United States); P. H. Fuoss, Materials Science Division, Argonne National Lab. (United States); G. B. Stephenson, Materials Science Division, Argonne National Lab. (United States) and Advanced Photon Source, Argonne National Lab. (United States); A. Robert, SLAC National Accelerator Lab. (United States); G. Grübel, Deutsches Elektronen-Synchrotron (Germany)

\section{LASERS}

8504 OL Pump-probe laser development for the European X-ray Free-Electron Laser facility (Invited Paper) [8504-20]

M. J. Lederer, M. Pergament, M. Kellert, European XFEL GmbH (Germany); C. Mendez, Univ. de Salamanca (Spain) 
8504 OM Spectral encoding based measurement of $x$-ray/optical relative delay to $\sim 10 \mathrm{fs} \mathrm{rms}$ [8504-21]

M. R. Bionta, D. French, SLAC National Accelerator Lab. (United States); J. P. Cryan, Lawrence Berkeley National Lab (United States); J. M. Glownia, SLAC National Accelerator Lab. (United States) and Stanford Univ. (United States); N. Hartmann, SLAC National Accelerator Lab. (United States); D. J. Nicholson, SLAC National Accelerator Lab. (United States) and Stanford Univ. (United States); K. Baker, Lawrence Livermore National Lab (United States); C. Bostedt, SLAC National Accelerator Lab. (United States); M. Cammarrata, Univ. de Rennes (France); M. Chollet, Y. Ding, D. M. Fritz, SLAC National Accelerator Lab. (United States); S. M. Durbin, Univ de Rennes (United States); Y. Feng, SLAC National Accelerator Lab. (United States); M. Harmand, Deutsches ElektronenSynchrotron (Germany); A. R. Fry, SLAC National Accelerator Lab. (United States); D. J. Kane, Mesa Photonics, LLC (United States); J. Krzywinski, H. T. Lemke, M. Messerschmidt, D. F. Ratner, S. Schorb, SLAC National Accelerator Lab. (United States); S. Toleikis, Deutsches Elektronen-Synchrotron (Germany); D. Zhu, W. E. White, R. N. Coffee, SLAC National Accelerator Lab. (United States)

8504 ON Applying field mapping refractive beam shapers to improve irradiation of photocathode of FEL [8504-23]

A. Laskin, V. Laskin, AdlOptica Optical Systems GmbH (Germany)

\section{DIAGNOSTICS II}

8504 OR X-ray photon diagnostics devices for the European XFEL [8504-27]

J. Grünert, J. Buck, C. Ozkan, W. Freund, S. Molodtsov, European XFEL GmbH (Germany)

8504 OS A hard x-ray transmissive single-shot spectrometer for FEL sources [8504-28]

Y. Feng, D. Zhu, J. M. Feldkamp, H. T. Lemke, A. Robert, D. M. Fritz, M. Cammarata, S. Lee, J. B. Hastings, J. L. Turner, SLAC National Accelerator Lab. (United States)

8504 OU Time-of-flight photoemission spectroscopy from rare gases for non-invasive, pulse-to-pulse $x$-ray photon diagnostics at the European XFEL [8504-30]

J. Buck, European XFEL GmbH (Germany); J. Viefhaus, F. Scholz, Deutsches Elektronen-

Synchrotron (Germany); M. Ilchen, European XFEL GmbH (Germany) and Deutsches Elektronen-Synchrotron (Germany); L. Glaser, Deutsches Elektronen-Synchrotron (Germany); C. Özkan, W. Freund, J. Grünert, S. Molodtsov, European XFEL GmbH (Germany)

POSTER SESSION

8504 OV Ultra-thin Bragg crystals for LCLS beam-sharing operation [8504-31]

Y. Feng, D. Zhu, A. Robert, H. T. Lemke, M. Chollet, D. M. Fritz, J. B. Hastings, J. M. Feldkamp, M. Cammarata, S. Moeller, SLAC National Accelerator Lab. (United States); M. Yabashi, K. Tono, SPring-8, RIKEN (Japan); X. Huang, Argonne National Lab. (United States) 
8504 OX Initial evaluation of the European XFEL undulator commissioning spectrometer with a single channel-cut crystal [8504-33]

C. Ozkan, W. Freund, European XFEL GmbH (Germany); J. Rehanek, Helmholtz-Zentrum Berlin für Materialien und Energie GmbH (Germany); J. Buck, European XFEL GmbH

(Germany); I. Zizak, Helmholtz-Zentrum Berlin für Materialien und Energie GmbH (Germany); J. Gruenert, European XFEL GmbH (Germany); F. Schaefers, A. Erko, Helmholtz-Zentrum Berlin für Materialien und Energie GmbH (Germany); S. Molodtsov, European XFEL GmbH (Germany)

Author Index 
Proc. of SPIE Vol. $8504850401-8$

Downloaded From: https://www.spiedigitallibrary.org/conference-proceedings-of-spie on 26 Apr 2023 Terms of Use: https://www.spiedigitallibrary.org/terms-of-use 


\section{Conference Committee}

Program Track Chair

Carolyn A. MacDonald, University at Albany (United States)

Conference Chairs

Stefan P. Moeller, SLAC National Accelerator Laboratory (United States)

Makina Yabashi, SPring-8/RIKEN (Japan)

Stefan P. Hau-Riege, Lawrence Livermore National Laboratory (United States)

Conference Program Committee

Henry N. Chapman, Deutsches Elektronen-Synchrotron (Germany)

Christian David, Paul Scherrer Institut (Switzerland)

Alan R. Fry, SLAC National Accelerator Laboratory (United States)

Gerhard Grübel, Deutsches Elektronen-Synchrotron (Germany)

Nina Rohringer, Max-Planck-Arbeitsgruppen für strukturelle Molekularbiologie (Germany)

Harald Sinn, European XFEL GmbH (Germany)

Kai Tiedtke, Deutsches Elektronen-Synchrotron (Germany)

Niels van Bakel, Nationaal instituut voor subatomaire fysica (Netherlands)

Session Chairs

1 Diagnostics I

Stefan P. Moeller, SLAC National Accelerator Laboratory (United States)

2 Optics 1

Christian David, Paul Scherrer Institut (Switzerland)

3 Detectors

Lothar Strueder, Max-Planck-Gesellschaft (Germany)

4 Applications

Adrian L. Cavalieri, Max Planck Research Department for Structural Dynamics (Germany) 
5 Lasers

Matthias Hoffmann, SLAC National Accelerator Laboratory (United States)

6 Optics II

Yiping Feng, SLAC National Accelerator Laboratory (United States)

7 Diagnostics II

Makina Yabashi, SPring-8/RIKEN (Japan) 


\section{Introduction}

The conference X-Ray Free-Electron Lasers: Beam Diagnostics, Beamline Instrumentation, and Applications was held for the first time in San Diego, California from August 13-16, 2012.

With the successful start-up and operation of the first hard X-ray free-electron laser (FEL), the Linac Coherent Light Source (LCLS) at Stanford in April of 2009, and, more recently, Japan's SPring-8 Angstrom Compact FEL, SACLA, in June of 2011, novel x-ray sources with unprecedented beam properties have become operational, offering great promise for exploring new scientific possibilities in ultrafast science with hard X-rays. These $4^{\text {th }}$ generation light sources operate in the Self Amplified Stimulated Emission (SASE) mode and provide radiation with wavelengths typically as short as about $1 \AA$ and pulses of unrivaled brightness, brevity, peak power density, and transverse coherence, exceeding the values of the same parameters as 3rd Generation synchrotron radiation sources by up to ten orders of magnitude. The implementation of this class of sources using superconducting linac technology, for example in the European XFEL in Germany (planned operation in ca. 2015) and the FLASH VUV FEL, successfully operating since 2005, is also expected to match or exceed the time-averaged power density generated by most present day sources by several orders of magnitude. Besides the aforementioned facilities, there are nearly a dozen other FELs currently being proposed or under construction. All are scheduled to become operational before 2020.

The rapidly growing user community utilizes these sources for experiments in areas ranging from atomic physics, material science, x-ray diffraction, and $x$-ray coherent imaging to high-energy-density physics. The experimental techniques are becoming increasingly complex posing great challenges for beam transport and beam shaping optics, diagnostics, and detectors as well as synchronization techniques with optical lasers for pump probe experiments. Interpretation of scientific results necessitates the development and refinement of beam pulse diagnostic techniques. New developments are already underway to advance specialized schemes to generate ultra-short pulses of a few femtoseconds, and various seeding techniques promising improved bandwidth and coherence properties that will involve novel directions in scientific applications.

This conference captured recent developments in this exciting field, spanning topics from new facilities, optical components, instrumentation, beam diagnostics to optical lasers, novel detectors, applications, and simulations.

We gratefully acknowledge the support of SPIE in adding this conference to their program, and thank them for their outstanding organization of the conference as well as to the editorial staff for their efforts in publishing the proceedings' volume. 
We would also like to thank the Program Committee for their support in many aspects of the program's organization, and the session chairs for their help in running the conference. Without the participants and their high-quality, scientific, and meaningful contributions, the success of 2012 meeting would not have been possible. Thank you.

Stefan Moeller Makina Yabashi Stefan Hau-Riege 